\title{
Does total joint arthroplasty impair erectile function?
}

\author{
Patrick Weber, Florian Schmidutz, Andreas Ficklscherer, Arnd Steinbrück, Volkmar Jansson, \\ Hans Roland Dürr
}

Department of Orthopedic Surgery, Physical Medicine and Rehabilitation, University Hospital, Ludwig-Maximilians-University (LMU), Munich, Germany

Submitted: 15 May 2016

Accepted: 12 June 2016

Arch Med Sci 2018; 14, 5: 1087-1092

DOI: https://doi.org/10.5114/aoms.2016.61939

Copyright @ 2016 Termedia \& Banach

\section{Abstract}

Introduction: Sexuality is an important factor in quality of life (QoL) and was reported to improve after total joint arthroplasty (TJA). However, one study group found evidence regarding a high rate of impaired erectile function of about $20 \%$ in male patients after TJA. As erectile dysfunction is a serious matter of concern and there are no data explaining this observation sufficiently, the purpose of this study was to revaluate the erectile function in patients after TJA.

Material and methods: All consecutive male patients scheduled for TJA were enrolled in this prospective study. Evaluation was performed pre- and 6 months postoperatively with the International Index of Erectile Function (IIEF-5) questionnaire. One hundred and fifty-nine patients fulfilled the inclusion criteria, and 51 patients wished not to take part in the study.

Results: From the 108 patients who were included prior to surgery, 101 (94\%) were available at the 6-month follow-up. In those patients, the preoperative erectile function was normal in $38(24.0 \pm 1.1)$, impaired in 45 $(14.7 \pm 6.5)$ and with no function in 18 patients. No significant difference in the pre- $(15.4 \pm 9.3)$ and postoperative $(15.3 \pm 9.2)$ IIEF-5 score was observed $(p=0.59)$ in the total group. Similarly, subgroup analysis revealed no significant difference in patients with total hip arthroplasty $(p=0.58)$, total knee arthroplasty $(p=0.37)$ or $>70$ years $(p=0.08)$.

Conclusions: The previously reported high rate of impaired erectile function after TJA of the lower extremity could not be confirmed. Total joint arthroplasty remains a safe procedure in terms of postoperative erectile function.

Key words: total knee arthroplasty, total hip arthroplasty, erectile dysfunction, sexuality.

\section{Introduction}

Total hip arthroplasty (THA) has been declared as the operation of the century [1]. This is related to the fact that total hip and knee replacement is a relatively safe procedure leading to a clear improvement in quality of life [2-6].

An important, but commonly barley discussed aspect after total joint arthroplasty (TJA) is sexual activity and erectile function. Although this is an important and relevant concern for most patients, surgeons often find it difficult to discuss this topic in a pro-active way $[7,8]$. This might also be related to the limited information available, as only a few studies have dealt with sexuality after TJA in the past. Furthermore, most studies

\author{
Corresponding author: \\ PD Dr. Patrick Weber MD \\ Department of \\ Orthopedic Surgery \\ Physical Medicine \\ and Rehabilitation \\ Campus Grosshadern \\ Ludwig-Maximilians- \\ University \\ Marchioninistr. 15 \\ 81377 Munich, Germany \\ E-mail: patrick.weber@ \\ med.uni-muenchen.de
}


focused on the coital position, as patients and especially women fear THA dislocation, but did not include sexual activity and erectile function after surgery $[9,10]$.

In the past, one study evaluated sexual function after total hip arthroplasty (THA) and reported improved function [11]. Recently, more studies have been focusing more specifically on sexual activity. Nunley et al. reported that patients under 60 years can expect to return to their preoperative baseline or even a higher level of sexual activity following TJA. They also found that most patients were more frequently sexually active with higher rates of satisfaction following their surgery [12].

In contrast to those positive results, a Danish study reported a high rate of sexual problems after TJA, especially in elderly men. Nordentoft et al. found that about $20 \%$ of male patients with preoperative normal erectile function (EF) had impairment of their EF postoperatively [13]. However, this finding could not be explained in a satisfactory manner, nor has it been validated.

Nevertheless, this observation remains a serious matter of concern, considering that in Germany about 350000 TJAs are performed annually. A rate of $20 \%$ erectile dysfunction after surgery would result in a rate of up to 35000 male patients experiencing new impairment of their EF after TJA [14, 15].

Therefore the aim of this study was to evaluate whether the high rate of erectile dysfunction can be reproduced in a German cohort. According to Nordentoft et al. we hypothesised that TJA leads to a significant change in the erectile function of male patients.

\section{Material and methods}

\section{Patients}

Between June 2008 and February 2010 all consecutive male patients scheduled for primary total hip (THA) or knee arthroplasty (TKA) were enrolled in the study. Exclusion criteria were patients younger than 18 years, previous open knee or hip surgery or prostatectomy. Furthermore, patients not able to participate to the 6-month follow-up or to complete the study questionnaire were excluded.

One hundred and fifty-nine patients fulfilled the inclusion criteria and were asked to participate in the study, with 51 refusing to participate due to personal reasons mainly related to their personal privacy. Finally, the study group consisted of 108 patients (Figure 1). The study protocol was approved by the local research ethics committee of our university and all patients enrolled gave written informed consent to take part in the study.

\section{Evaluation of erectile function}

Erectile function was assessed with the standardised and established International Index of Erectile Function (IIEF-5) questionnaire. The IIEF-5 is a self-assessment questionnaire consisting of 5 questions, four rating between 0 and 5 points and one rating between 1 and 5 points. This results in a total score between 1 and 25 points, with higher scores representing better erectile function. Patients with a score of 25 to 22 are rated as having normal EF [16].

\section{Statistical analysis}

Sample size calculation was performed prior to the beginning the study with power analy-

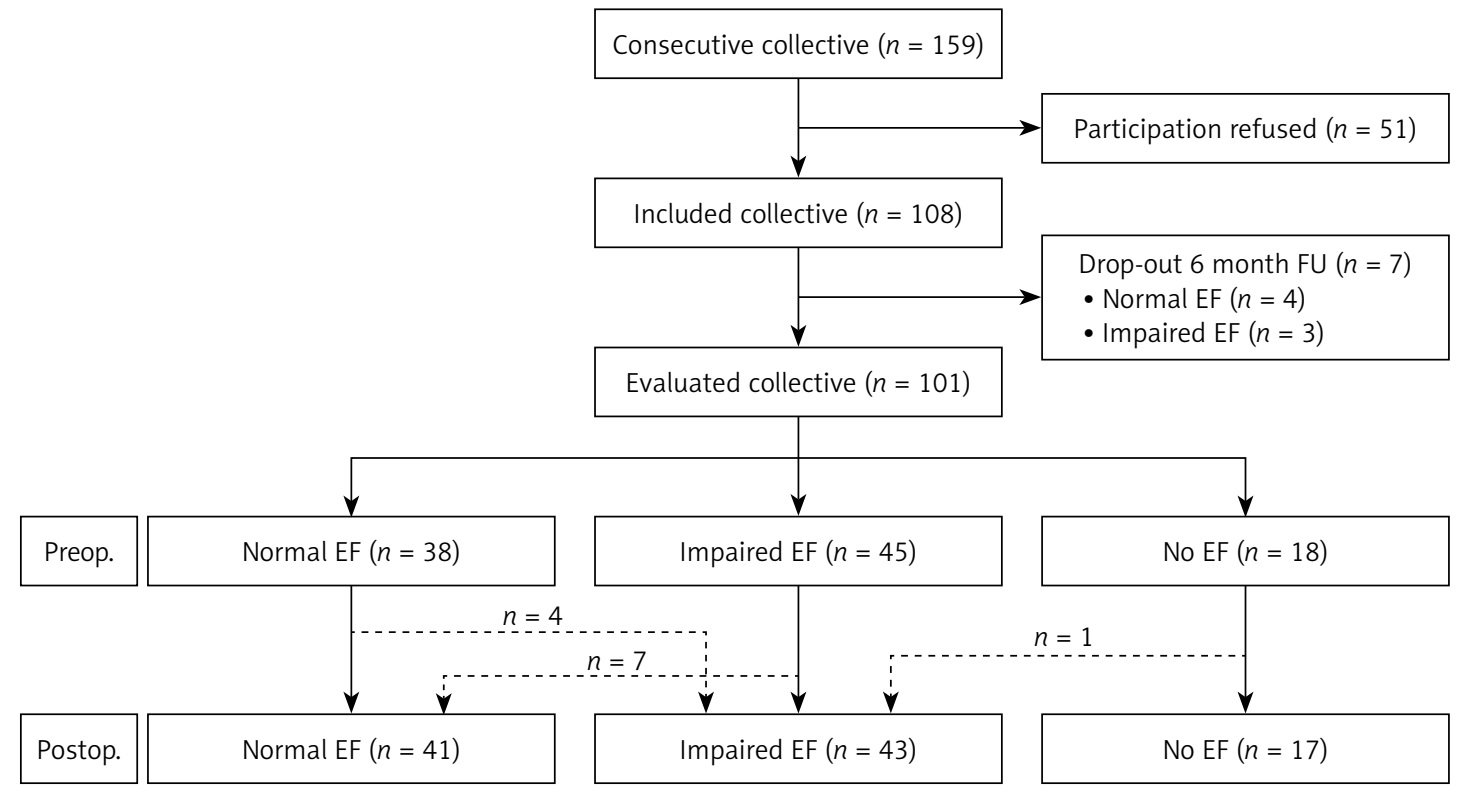

Figure 1. Patient flow diagram to evaluate erectile dysfunction after TJA 
ses. To show a difference of 3 points in the IIEF-5 (standard deviation: 10) before and after surgery, 89 patients were required, giving a power of $80 \%$ ( $\alpha$ level of 0.05 and $\beta$ level of 0.2 ). Defining a maximal acceptable drop-out rate of $20 \%$, it was estimated that 107 patients needed to be included in this study.

Descriptive statistics was performed and demographic data are given as mean and range. The results of the IIEF-5 are given as mean and standard deviation (SD). Statistical analysis was performed using the SPSS software package (SPSS, Chicago, Illinois). The paired Student's $t$-test for normal variable parameters was applied to compare the pre- and postoperative outcome scores between the groups. A p-value of $<0.05$ was considered statistically significant.

\section{Results}

From the 108 patients included, 101 (94\%) were available for the 6-month follow-up. Seven patients were lost to follow-up, one due to a newly diagnosed tongue cancer receiving radio-chemotherapy, and the other 6 patients could not be contacted or refused to participate further. Patients who could not be followed up had a normal $(n=4)$ and impaired $(n=3)$ EF (Figure 1$)$.

From the finally evaluated 101 patients, 38 had preoperative normal erectile function (IIEF score $24 \pm 1.1$ ) (IIEF-5 $\geq 22$ ), 45 had impaired erectile function (IIEF score: $15.4 \pm 9.3)(\mathrm{IIEF}-5<22$ ) and 18 had no erectile function before surgery (IIEF score: $1 \pm 0$ ) (Table I). This gives a preoperative prevalence of normal erectile function of $38 \%$ and a prevalence of erectile dysfunction of $62 \%$.
After surgery, there was a shift of 4 patients from the group with normal EF to the group with impaired $(n=3)$ function. On the other hand, there was a shift of 7 patients from the impaired group to the normal group. One patient shifted from the group of no function to the group of impaired function.

Postoperative evaluation of the 101 patients revealed that 41 had normal erectile function (minimum IIEF-5 score: 22 ), 43 had impaired erectile function (IIEF-5 score: < 22) and 17 had no erectile function. This gives a postoperative prevalence of normal erectile function of $41 \%$ and a prevalence of erectile dysfunction of $59 \%$.

Comparing the IIEF-5 score of the total group revealed a preoperative score of $15.4 \pm 9.3$, which was not significantly different from the postoperative score of $15.3 \pm 9.2(p=0.59)$ (Table II).

Similarly, when excluding the 18 patients who had no pre- or postoperative erectile function, and only analysing the 83 patients with a normal or impaired $E F$, no significant difference was found (19.0 \pm 6.7 vs. $18.6 \pm 7.1, p=0.46)$.

Similarly, no significant difference was observed pre- or postoperatively for only the patients with normal EF (Table II).

\section{Subgroup analysis}

THA

From the 101 patients, 67 (67\%) underwent THA. The indication for THA was primary osteoarthritis in 58 (87\%), avascular necrosis in $6(9 \%)$, and osteoarthritis due to dysplasia in 3 (4\%) patients. In 46 (69\%) cases cementless THA, in $16(24 \%)$ cases hybrid THA, in $4(6 \%)$ cases fully

Table I. Demographic characteristics of patients and subgroup analysis to evaluate erectile dysfunction after total joint arthroplasty (TJA)

\begin{tabular}{|lcccc|}
\hline Parameter & $\begin{array}{c}\text { Total group } \\
N=101\end{array}$ & $\begin{array}{c}\text { Normal EF } \\
N=38\end{array}$ & $\begin{array}{c}\text { Impaired EF } \\
N=45\end{array}$ & $\begin{array}{c}\text { No EF } \\
N=18\end{array}$ \\
\hline Age [years] & $62.2(29.5-86.6)$ & $61.3(30.2-78.0)$ & $59.6(29.5-76.6)$ & $76.2(61.6-86.6)$ \\
\hline Height $[\mathrm{cm}]$ & $176.5(160-196)$ & $175.8(163-187)$ & $177.8(160-196)$ & $174(168-183)$ \\
\hline Weight $[\mathrm{kg}]$ & $85.3(49-173)$ & $87.1(73-108)$ & $88.1(68-115)$ & $72.5(49-83)$ \\
\hline ASA score & $2.3(1-4)$ & $2.1(1-3)$ & $2.3(1-4)$ & $2.9(2-3)$ \\
\hline
\end{tabular}

Table II. Pre- and postoperative erectile function in a cohort of patients after total joint arthroplasty (TJA)

\begin{tabular}{|lccc|}
\hline Variable & Preop. IIEF-5 & Postop. IIEF-5 & $P$-value \\
\hline Total group $(n=101 / n=101)$ & $15.4 \pm 9.3$ & $15.3 \pm 9.2$ & 0.59 \\
\hline Normal function $(n=38 / n=41)$ & $24.0 \pm 1.1$ & $23.2 \pm 8.58$ & 0.07 \\
\hline Impaired function $(n=45 / n=43)$ & $14.7 \pm 6.5$ & $14.6 \pm 6.3$ & 0.92 \\
\hline No function $(n=18 / n=17)$ & $1 \pm 0$ & $1.5 \pm 2.1$ & 0.33 \\
\hline Normal and impaired cohort $(n=83 / n=84)$ & $19.0 \pm 6.7$ & $18.6 \pm 7.1$ & 0.46 \\
\hline
\end{tabular}


Table III. IIEF-5 of the patients in the subgroup analysis after total joint arthroplasty (TJA)

\begin{tabular}{|lccc|}
\hline Variable & Preop. IIEF-5 & Postop. IIEF-5 & $P$-value \\
\hline THA $(n=67)$ & $17.5 \pm 8.7$ & $15.5 \pm 8.4$ & 0.58 \\
\hline TKA $(n=34)$ & $11.8 \pm 9.2$ & $11.0 \pm 9.4$ & 0.37 \\
\hline$>70$ years $(n=29)$ & $10.5 \pm 10.5$ & $9.2 \pm 10.3$ & 0.08 \\
\hline
\end{tabular}

cemented THA, and in 1 (1\%) patient hip resurfacing arthroplasty was performed. The subgroup analysis for patients with THA did not reveal a significant difference in the pre- and postoperative IIEF-5 score (Table III).

\section{TKA}

From the 101 patients 34 (33\%) underwent TKA The reason for TKA was primary osteoarthritis in all patients, and cemented TKA was performed in all cases. The subgroup analysis for patients with TKA did not reveal a significant difference in the pre- and postoperative IIEF-5 score (Table III).

\section{Age}

From the 101 patients, 29 were aged $>70$ years and $72<70$ years. Subgroup analysis for this age group did not reveal a significant difference in the pre- and postoperative IIEF-5 score (Table III).

\section{Discussion}

The aim of this study was to determine whether the high rate of erectile dysfunction after TJA can be reproduced in a German cohort. Our hypothesis was that TJA leads to a significant change in the erectile function of male patients.

The results of our findings do not confirm the observation of Nordentoft et al. of an increased rate of impaired erectile function in males after undergoing TJA [13]. In our German cohort the performance of TJA of the lower limb did not result in a significant change in postoperative erectile function compared to the preoperative baseline status.

This appears important, as a study from Lavernia et al. showed that $82 \%$ of patients with arthritis of the hip still were sexually active [8]. Moreover, sexuality in the elderly should be considered an important part of their life quality, and more than $70 \%$ of men aged between 70 and 80 years are reported to be sexually active [17].

On the other hand, erectile dysfunction is a growing problem in the elderly population. In this context, the prevalence of erectile dysfunction in our male study cohort was found to be about $62 \%$ and might appear high. However, those numbers in our male group with the age of about 63 years are comparable to the rates reported in the current literature, as in the US, the prevalence of erectile dysfunction in men aged between 40 and 70 years was reported to be $52 \%$ [18] and in all patients over 60 years about 53.4\% [17] Similarly, in France the prevalence in men aged over 40 years was reported to be $31.6 \%$ [19].

The etiology of erectile dysfunction is multifactorial and usually involves vascular, anatomic, neurologic, endocrinological, and psychological factors [20]. Up to now, TJA has not been considered as a common risk factor in the literature. However, the study by Nordentoft et al. which reported an increase of about 20\% after TJA [13] was a matter of concern, as this would indicate that about 35000 male patients in Germany would annually experience erectile dysfunction related to TJA. As patients expect better quality of life and at least a return to their preoperative sexual function after TJA, a significant increase in erectile dysfunction would represent a serious problem [7, 8]. However, the data from this study in a German male cohort did not find an increase in risk of experiencing erectile dysfunction related to TJA.

Certainly there is an annually increasing risk in patients over 60 years of experiencing erectile dysfunction, but as already pointed out by Nordentoft et al., this is about 3\% a year and not commonly around 20\% [13].

The authors also discussed the impact of TJA as major surgery which might affect the sexual function and erectile function. However, they referred this to data from heart transplantation or limb amputation [13, 21, 22]. Considering that THA or TKA is commonly performed within 60$90 \mathrm{~min}$ and causes blood loss of usually $500 \mathrm{ml}$ in THA and $300 \mathrm{ml}$ in TKA, it appears unlikely that this results in impaired sexual function. Furthermore, in contrast to surgery of the prostate or inguinal hernia repair, TJA usually does not affect vessels or nerves which are directly related to sexual function.

Also a subgroup analysis of patients with TKA or THA or over 70 years old did not reveal an increase of impaired EF after surgery. Therefore, we do not think that TJA should be considered a risk factor of erectile dysfunction.

This is further underlined by several studies which have mainly reported better sexual function after TJA. The improvement is mainly related to less pain and an improved range of motion of the 
joint $[10,12]$. Yoon et al. investigated the satisfaction after TJA and reported no change in $68.8 \%$, greater satisfaction in $23.4 \%$ and less satisfaction only in $7.9 \%$. Lower satisfaction was referred to the fear of dislocation and a higher stress level [10]. Furthermore, a recent US multicentre study in young patients below 60 years that included 806 THA and 542 TKA as well as 181 control patients did not find a significant difference in sexual activity following TJA [12]. In this study only $1.3 \%$ of THA and $1.6 \%$ of TKA patients stated that they were not sexually active which was directly related to their operation. Again one of the main reasons was related to instability of the joint [12].

Although none of those studies specifically address EF as an outcome parameter, we assume that an erectile dysfunction rate of about $20 \%$ in male patients would clearly have affected the sexual activity in those studies. As almost all studies reported about the same or even an improved sexual activity level postoperatively, we believe that this confirms our results showing no significant deterioration, but rather a slight improvement in the erectile function related to the TJA of the lower extremity.

This study has several limitations. First, discussion about erectile function is an uncomfortable topic for most patients. This is illustrated by the fact that 51 (31\%) from 159 patients refused to participate. Therefore the study only included patients willing to participate. Nevertheless, the preoperative power analysis revealed that 89 patients were required, and even after a drop out a final cohort of 101 patients completed the study. So the study seems not to be underpowered.

Second, EF is an intimate subject bearing a risk of bias, especially in men with a tendency to underreport problems. Therefore, in this study, patients completed the IIEF-5 without the attendance of a physician or a paramedical person. This should lead to more honest reporting and should have minimized the tendency of underreporting. Furthermore, the preoperative results match well with postoperative results, indicating relatively valid reporting of the EF.

Thirdly, we did not obtain more specific information such as the absolute number of sexual contacts per week, duration or kind of sexual activity, which could provide even more detailed information. However, we assumed that this would have increased the refusal and drop-out rates enormously.

Fourthly, we only followed the patients for 6 months, which is a relatively short follow-up in TJA. However, in patients of that age, there is a risk that they will develop erectile dysfunction due to reasons not related to surgery. This is the reason we chose this short interval after surgery.
In conclusion, this study showed that TJA of the lower extremity did not lead to impaired erectile function. Total joint arthroplasty remains a safe procedure in terms of EF, and most patients can expect at least a stable or even a slight improvement in erectile function after TJA.

\section{Conflict of interest}

The authors declare no conflict of interest.

\section{References}

1. Learmonth ID, Young C, Rorabeck C. The operation of the century: total hip replacement. Lancet 2007; 370: 1508-19.

2. Garbuz DS, Xu M, Sayre EC. Patients' outcome after total hip arthroplasty: a comparison between the Western Ontario and McMaster Universities index and the Oxford 12-item hip score. J Arthroplasty 2006; 21: 9981004.

3. Mainard D, Guillemin F, Cuny C, Mejat-Adler E, Galois L, Delagoutte J. Quality of life assessment one year after total hip or knee arthroplasty [French]. Rev Chir Orthop Reparatrice Appar Mot 2000; 86: 464-73.

4. Knahr K, Kryspin-Exner I, Jagsch R, Freilinger W, Kasparek $M$. Evaluating the quality of life before and after implantation of a total hip endoprosthesis [German]. Z Orthop Ihre Grenzgeb 1998; 136: 321-9.

5. Czyzewska A, Glinkowski WM, Walesiak K, Krawczak K, Cabaj D, Gorecki A. Effects of preoperative physiotherapy in hip osteoarthritis patients awaiting total hip replacement. Arch Med Sci 2014; 10: 985-91.

6. Ficklscherer A, Stapf J, Meissner KM, et al. Testing the feasibility and safety of the Nintendo Wii gaming console in orthopedic rehabilitation: a pilot randomized controlled study. Arch Med Sci 2016; DOI 10.5114/ aoms.2016.59722.

7. Christofilopoulos P, Charbonnier C. CORR insights: high rates of interest in sex in patients with hip arthritis. Clin Orthop Relat Res 2016; 474: 300-1.

8. Lavernia CJ, Villa JM. High rates of interest in sex in patients with hip arthritis. Clin Orthop Relat Res 2016; 474: 293-9.

9. Charbonnier C, Chague S, Ponzoni M, Bernardoni M, Hoffmeyer P, Christofilopoulos P. Sexual activity after total hip arthroplasty: a motion capture study. J Arthroplasty 2014; 29: 640-7.

10. Yoon BH, Lee KH, Noh S, Ha YC, Lee YK, Koo KH. Sexual activity after total hip replacement in Korean patients: how they do, what they want, and how to improve. Clin Orthop Surg 2013; 5: 269-77.

11. Stern SH, Fuchs MD, Ganz SB, Classi P, Sculco TP, Salvati EA. Sexual function after total hip arthroplasty. Clin Orthop Relat Res 1991; 269: 228-35.

12. Nunley RM, Nam D, Bashyal RK, et al. The impact of total joint arthroplasty on sexual function in young, active patients. J Arthroplasty 2015; 30: 335-40.

13. Nordentoft T, Schou J, Carstensen J. Changes in sexual behavior after orthopedic replacement of hip or knee in elderly males: a prospective study. Intern J Impot Res 2000; 12: 143-6.

14. Siebert HR. Knie- Totalendoprothesen: Erstimplantation. In: GmbH A-IfaQuFiG (ed.) Qualitätsreport 2011. Göttingen 2012; 160-5. 
15. Siebert HR. Knie- Endoprothesen: Wechsel und Komponentenwechsel. In: GmbH A-IfaQuFiG (ed). Qualitätsreport 2011. Göttingen 2012; 166-9.

16. Rosen RC, Cappelleri JC, Smith MD, Lipsky J, Pena BM. Development and evaluation of an abridged, 5-item version of the International Index of Erectile Function (IIEF-5) as a diagnostic tool for erectile dysfunction. Intern J Impot Res 1999; 11: 319-26.

17. Braun M, Wassmer G, Klotz T, Reifenrath B, Mathers M, Engelmann U. Epidemiology of erectile dysfunction: results of the 'Cologne Male Survey'. Intern J Impot Res 2000; 12: 305-11.

18. Feldman HA, Goldstein I, Hatzichristou DG, Krane RJ, McKinlay JB. Impotence and its medical and psychosocial correlates: results of the Massachusetts Male Aging Study. J Urol 1994; 151: 54-61.

19. Giuliano F, Chevret-Measson M, Tsatsaris A, Reitz C, Murino M, Thonneau P. Prevalence of erectile dysfunction in France: results of an epidemiological survey of a representative sample of 1004 men. Eur Urol 2002; 42: 382-9.

20. Lizza EF, Rosen RC. Definition and classification of erectile dysfunction: report of the Nomenclature Committee of the International Society of Impotence Research. Intern J Impot Res 1999; 11: 141-3.

21. Williamson G, Walters A Perceived impact of limb amputation on sexual function: a study of adult amputees. J Sex Res 1996; 33: 221-30.

22. Mulligan T, Sheehan H, Hanrahan J. Sexual function after heart transplantation. J Heart Lung Transplantation 1991; 10: 125-8. 